ISSN: $1130-3743$

\title{
PENSAR SOBRE LA EDUCACIÓN DESDE UNA CONCEPCIÓN SISTÉMICO-CIBERNÉTICO
}

\section{THINKING ABOUT EDUCATION FROM A SYSTEMIC-CYBERNETIC PERSPECTIVE}

\author{
BEGOÑA GROS SALVAT \\ Universitat de Barcelona. Facultad de Ciencias de la Educación. Departament de Teoria $i$ \\ Historia de la Educación. Paseo Vall d'Hebron, 171. Edificio Llerant. O8028 Barcelona.
}

A la memoria del Doctor Alejandro Sanvisens.

RESUMEN

El objetivo princial de este artículo es mantener que la teoría sistémico-cibernética proporciona un importante bagaje para el estudio de la educación.

Durante los años 60 y 70 la contribución de estas teorías desempeñó un importante papel en el desarrollo de una nueva concepción de la Pedagogía basada en la importancia de contemplar la realidad con un todo, como un sistema. Más tarde, autores como Morin, von Foerster, Glasersfeld, Prigogine, Maturana, etc. introducen una nueva aproximación llamada "segunda cibernética"

Entendemos que la segunda cibernética introduce tres ideas que son muy importantes para el estudio de los procesos educativos: la idea de subjetividad en el conocimiento científico, una aproximación constructivista y la idea de complejidad.

SUMMARY

The main goal of this article is to maintain that the theory of systems and the cybernetics provide an important background to the study of education.

During the 60's and 70's the contribution of these theories had an important role in the development of a new conception of pedagogy based on the importance of looking reality as a whole, as a system. Later, authors like Morin, von Foerster, Glasersfeld, Prigogine, Maturana, etc., introduce a new approach called "the second cybernetic". 
We consider that the second cybernetic introduce three ideas that are very important for the study of any educational process: The idea of subjectivity in the sccientific knowledge, a constructivistic approach and the idea of complexity.

INTENCIONES

"No es mi obligación entregar a los demás lo objetivamente mejor, sino lo mío, tan pura y sinceramente como sea posible.

(HESSE, H.)

Quisiera comenzar este artículo explicando las razones que me han llevado a realizarlo. Estas son básicamente de índole personal. A pesar de ello, considero que su explicitación puede ayudar a comprender mejor las ideas que mantendré a lo largo del mismo.

Entre en contacto con la teoría cibernética cuando estudiaba pedagogía en la Universidad de Barcelona a través de las clases del Dr. Alejandro Sanvisens. El conocimiento de la cibernética, la teoría de sistemas así como de la epistemología piagetiana constituyeron tres pilares teóricos muy importantes en mi formación. Posteriormente al convertirme en profesora de las asignaturas de Pedagogía Cibernética y Teoría del aprendizaje, se me dio la oportunidad de tenerlas que transmitir a otros estudiantes. Durante este proceso, la concepción sistémico-cibernética con la que yo había comenzado a trabajar entró en crisis. Algunos aspectos me parecían sumamente interesantes desde el punto de vista teórico pero limitados para afrontar una aplicación práctica. El estudio de las teorías de la complejidad me abrieron nuevos caminos, pero las dudas todavía persistían. El elevado grado de abstracción de la mayoría de las aportaciones de los autores me hacía reconsiderar su valor en el ámbito pedagógico. Estaba tan preocupada por este problema que tardé bastante tiempo en darme cuenta de un hecho que se repetía sistemáticamente en las clases de esta asignatura. Para los alumnos ${ }^{1}$, la concepción cibernética y sistémica les suponía un replanteamiento de muchas de sus concepciones sobre la educación. De este modo, la importancia que tiene el paso de una concepción lineal a una visión circular, de una concepción determinista a una concepción sistémica sólo fuí capaz de apreciarlo a través de las aportaciones que los alumnos realizaban. Desde el momento en que me dí cuenta de que en cierta manera había un antes y un después en la forma de pensar, en la forma de mirar la realidad, comencé a explorar esta idea de forma más consciente y a darme cuenta de su importancia. Cambiar la forma de mirar la realidad es también una forma de replantearse la propia práctica.

Es justo decir que las ideas que a cotinuación expondré surgen, sobre todo a partir de las palabras y los escritos de los estudiantes. No los hago responsables de las ideas que mantendré en este artículo pero sé que sin ellos, éste nunca habría sido escrito de esta forma.

1. La asignatura de Pedagogía Cibernética en la Facultad de Pedagogía de la Universidad de Barcelona ha sido obligatoria para los alumnos del último curso de la especialidad de Pedagogía Sistemática y en los nuevos planes de estudio tiene carácter de materia optativa de segundo ciclo. 
Las posiciones que sostendré a lo largo de este artículo girarán en torno a cinco ideas:

1. Durante el periódo de formación de los profesionales de la educación es necesario dotar de una buena base teórica que capacite para actuar y pensar sobre las actuaciones. En mi opinión, en la formación actual se ha ido perdiendo el aprendizaje, la reflexión sobre teorías "fuertes". Se adquieren ideas cada vez más fragmentadas y sin hilazón, lo que produce inseguridad para enfrentarse con la propia realidad.

2. Existen diversas teorías que yo misma podría mantener como válidas para comenzar el período de formación. Sin embargo, me centraré sólo en una aportación: la perspectiva sistémico-cibernética. Consideraré que la teoría cibernética y la teoría de sistemas proporcionan una serie de herramientas conceptuales que permiten tener una visión más completa de la educación.

3. No creo que la teoría cibernética y sistémica sean las únicas teorías que deban estudiarse. Proporcionan una forma de ver la realidad más interesante que las teorías deterministas y mecanicistas clásicas. Sin embargo, su estudio debe completarse con otras teorías más centradas en el ámbito educativo, más estrechamente ligadas a la práctica educativa.

4. No todas las aportaciones de la cibernética son igualmente válidas. En este sentido, trataré de mostrar cómo las aportaciones de la cibernética de segundo orden y las teorías de la complejidad son las que proporcionan una visión más interesante de lo educativo y lo social.

5. Si bien la perspectiva cibernética y sistémica permiten desarrollar un pensamiento más complejo sobre la educación, no ha proporcionado hasta el momento una metodología a plicativa. No se derivan prácticas cibernéticas o sistémicas de forma directa lo que, desde mi punto de vista, es un aspecto importante a considerar aunque ello no invalida su utilización a nivel formativo.

\section{APRENDER A MIRAR DE OTRA MANERA}

"Comprender no es descubrir hechos, ni extraer inferencias lógicamente válidas de premisas aceptadas, sino que consiste en adoptar el punto de vista adecuado desde el cual "ver" el chiste, oír la expresión de la música o ver la manera de salir de la niebla filosófica" (WITTGENSTEIN, L.)

A. Karmiloff-Smith y Bärbel Inhelder (1984) en un estudio experimental sobre la evolución del pensamiento en los niños mantenían una interesante idea; los niños elaboran continuamente teorías sobre el funcionamiento del mundo que contrastan con su propia práctica. Estas autoras publicaron el resultado de sus investigaciones en un artículo cuyo título nos da la clave de su tesis "si quieres avanzar, hazte con una teoría".

Si pensamos en nuestra vida cotidiana nos daremos cuenta como de forma constante desarrollamos teorías que nos explican e incluso justifican nuestras propias acciones o las de los demás. En este sentido, cuando miramos no vemos las 
cosas y luego las interpretamos sino que vemos desde el propio significado. Ver, oir, escuchar, no son operaciones pasivas, sino exploraciones activas, más o menos conscientes, a través de las cuales extraemos las informaciones que nos interesan.

Cuando una persona comienza a formarse en el ámbito de la pedagogía puede encontrarse con nombres de autores, conceptos, términos, técnicas educativas, etc., totalmente novedosas. No obstante lo que es evidente es que todos los estudiantes de pedagogía tienen ideas sobre qué es la educación. Muchas veces me he cuestionado si estas ideas previas cambian durante su formación. Evidentemente no tengo la respuesta. Sin embargo, sí recuerdo a algunos estudiantes con los cuales resulta dificil mantener un diálogo pues poseían ideas tan definitivas que todo era reinterpretado desde su visión particular, dificultando su propio enfrentamiento con nuevas ideas.

El conocimiento pedagógico y, desde luego, la acción educativa está impregnada de creencias, ideologías, valores morales, etc. En definitiva, de ideas que aparezcan en nuestra propia sociedad. Los teóricos de la pedagogía no las deberían ni rechazar ni olvidar. Si algo puede aportar la Pedagogía, desde su perspectiva académica, es la clarificación de la validez de muchas de estas creencias así como la sistematización y profundización de las diversas perspectivas. En este sentido, cuando un estudiante entra en la facultad de Pedagogía debería ocurrir "algo". Debería verse enfrentado con sus propias teorías, con sus propias ideas, debería producirse un cierto desorden interno. Nada traumático, desde luego, pero que le permitiera reconstruir su propia visión ofreciéndole además herramientas para sistematizar y profundizar sus propias creencias. Esta perspectiva, que no es otra que la de un planteamiento constructivista del conocimiento es muy dificil de llevar a la práctica. Sin embargo, considero que un estudio en profundidad de teorías "fuertes" que proporcionen herramientas conceptuales para pensar sobre la educación es una forma o camino interesante a explorar.

La teoría cibernética y la teoría de sistemas poseen un elevado grado de abstracción y este hecho tiene sus ventajas y sus limitaciones. La principal ventaja es que ofrecen una utilización conceptual transdisciplinar y permiten aprender a mirar la realidad desde una perspectiva más completa. Su mayor limitación es la carencia de aplicaciones pedagógicas basadas en este planteamiento. Por ello, el planteamiento sistémico-cibernético no puede fundamentar toda la formación pedagógica, necesariamente ha de ser complementado con otras perspectivas.

\section{LA CIBERNÉTICA DE LOS SISTEMAS OBSERVADOS}

"Hija: Dijiste que nosotros sólo hacemos las partes de un todo. Padre: No, dije que las partes son útiles, cuando queremos describir los todos.

Hija: Así, ¿tú quieres describir los todos? Pero, .cuando lo hayas hecho ¿que harás?». (BATESON, G. 1979).

El nacimiento de la cibernética se sitúa en 1948 cuando Norbert Wiener pública la obra Cibernetics definiendo a esta nueva disciplina como "la teoría del control 
y la información tanto en el animal con en la máquina” (Wiener 1971, 34 versión castellana). Wiener intentó realizar una especie de ciencia resumen, de ciencia síntesis donde se unificaran dominios muy diversos (físicos, biológicos, matemáticos, etc). Así «la cibernética se afirma como revolución epistemológicca al replantear la idea de objeto no como una unidad discreta sino en el nivel organizacional y superior de unidad compleja e interrelacionada" (Morin-Wiener, 1976, 61).

La teoría cibernética desarrolla una teoría del control que va a otorgar una visión nueva del funcionamiento de la realidad. Cinco conceptos de esta teoría supondrán una ruptura con respecto a la concepción de la ciencia clásica:

\section{El concepto de máquina}

Durante la II Guerra Mundial, a N. Wiener se le encomendó la creación de mecanismos de control para la artillería antiaérea que tuvieran la capacidad de regular su propia trayectoria. Este diseño le condujo al estudio del proceso de regulación de los organismos vivos y lo que Wiener hizo es extraer los datos que sobre biología se poseían y aplicarlos al diseño de la máquina. Es este dato muy relevante pues la comparación funcional entre la mente y la máquina que posteriormente incorporó la psicología cognitiva y la inteligencia artificial encuentran aquí sus orígenes. Pero también es importante porque hasta aquel momento no había habido ninguna relación entre los estudios biológicos y el diseño de máquinas, aspecto presente en la actualidad tanto en el diseño de hardware como del software.

Al principio, el interés de la cibernética se situó en el diseño de máquinas. Sin embargo, el propio Wiener amplió dicho objetivo y lo extendió al entendimiento del funcionamiento de sistemas humanos y sociales (Wiener, 1969). Del diseño de máquinas se pasaba al análisis de "máquinas" ya existentes, que no necesariamente han sido organizadas por nosotros pero de las cuales formamos parte.

\section{La noción de circularidad}

La cibernética introduce la idea de circularidad a través del concepto de retroalimentación o feedback. El feedback se define como la capacidad de respuesta para el mantenimiento de un estado de equilibrio. El feedback es pues un mecanismo que conduce a la regulación de un sistema. La regulación se produce siempre tras la ruptura de equilibrio. Es decir, cuando el estado ideal del sistema no coincide con su estado actual. En este caso, el sistema reacciona produciéndose una nueva búsqueda de equilibrio.

La utilización del concepto de feedback rompe la idea de causalidad tradicional en la que los efectos se encadenan de forma lineal. Por el contrario, el concepto de feedback conduce a la descripción de procesos circulares.

La idea de circularidad desarrollada por Wiener se centra en el feedback negativo. La reacción del sistema tiene un efecto contrario al del elemento perturbador 
y, por consiguiente, éste busca recuperar el equilibrio a través del mantenimiento del estado ideal. En definitiva, es este un feedback de tipo "conservador" ya que la meta o estado ideal del sistema se mantiene constante.

En 1968, Maruyama introdujó el concepto de feedback positivo que, a diferencia del negativo, amplifica la desviación. Este tipo de retroacción conduce a una desviación cada vez mayor respecto al estado ideal. Por este motivo, el feedback positivo podría llevar a la destrucción del sistema. No obstante, puede también convertirse en un mecanismo de creación de nuevos sistemas o de reestructuración de los ya existentes. La utilización de este concepto ha permitido explicar la evolución de los sistemas sociales y humanos en los cuales se complementan y entrecuzan los dos tipos de retroacciones.

\section{La noción de información}

La información es para la teoría cibernética un elemento fundamental para la organización del sistema. Lo que el enlace circular de los componentes retroalimenta no es sólo materia y energía, sino que hay allí un proceso informacional y organizacional.

Determinar el significado del término información, a pesar de su clara importancia, sigue siendo muy problemático. Como afirma Morin "no podemos decir casi nada acerca de ella, pero tampoco podemos prescindir de ella" $(1994,47)$.

El concepto de información utilizado por la cibernética tiene su origen en la teoría comunicativa de Shannon y Weaver que trataba de la transmisión de mensajes y actualmente está integrada en la teoría de la comunicación. Esta noción de información está basada en la medida de la información a través de unidades elementales de información o bits (binary digits), muy utilizados en la actualidad en la informática y las telecomunicaciones. A esta dimensión de la información, la cibernética le añade un sentido organizacional. De hecho, un "programa" portador de información lo que hace es comunicar un mensaje a una máquina que computa un cierto número de operaciones.

La noción de información de la teoría cibernética se elabora también a partir de la termodinámica. El segundo principio de la termodinámica había sido formulado mediante una ecuación de probabilidad que expresaba la tendencia de los sistemas a la entropía, es decir, al desorden, a la desorganización. Al mismo tiempo, las ecuaciones utilizadas por la teoría de la comunciación de Shannon y Weaver mostraban cómo la entropía crece en un sentido inverso a la información De aquí se dedujo que hay una equivalencia entre la información y la entropía negativa o neguentropía. En definitiva, que la información es una medida de la organización, del orden.

El problema del uso de la noción de información desde este punto de vista es su gran ambigüedad. De hecho el aspecto comunicacional tal y como se presenta en esta teoría no da cuenta del sentido de la información ni de la estructura de los mensajes. Es pues, desde mi punto de vista, uno de los conceptos que presenta más lagunas y que precisa un mayor estudio. Desde luego, no es éste un problema que se sitúa de forma exclusiva en la teoría cibernética. Cada vez se 
habla más de información pero, en cambio, los límites entre el conocimiento, el saber, los datos, la información, etc. resultan discutibles y borrosos.

En definitiva, la aportación ofrecida por la perspectiva cibernética que considero del mayor interés es la de concebir la información desde un punto de vista organizacional.

\section{La noción del control se complejiza a través de la noción de regulación.}

Fijar la trayectoria de un sistema no es imponer un camino directo y predictible sino establecer ciertas restricciones, reestructurar constantemente los diferentes elementos del sistema. Regular es, en definitiva, generar diversos niveles de estabilidad como resultado de un cambio constante en otros niveles del funcionamiento del sistema. De este modo, el control está en función de los procesos de regulación.

\section{Los sistemas explicables en términos de circularidad son sistemas donde la evolución es un factor muy importante.}

El equilibrio homeostático de un sistema es importante pero las progresivas equilibraciones del sistema solo pueden explicarse a través de las regulaciones efectuadas a lo largo del tiempo. Este aspecto procesual es un elemento clave dentro de la teoría de la cibernética y en su aplicación pedagógica se utilizará para resaltar el carácter procesual de los fenómenos educativos.

La teoría de sistemas elaborada por Bertalanffy contribuyó también al propio desarrollo de la cibernética. De hecho, las relaciones entre ambas teorías han sido siempre muy estrechas hasta el punto de que, en ocasiones, se hace muy difícil diferenciarlas. En principio, el campo de la teoría de sistemas es más amplio porque en un sentido propugna que toda realidad conocida puede ser concebida como un sistema. Por este motivo, A. Jdanko (1990) ha propuesto refundir ambas teorías en una sola proponiendo la denominación de teoría de sistemas cibernéticos.

La teoría de sistemas que empezó con Bertalanffy a partir de sus reflexiones sobre la situación de los estudios biológicos se expandió rápidamente a partir de los años 50 en las más variadas áreas (física, economía, sociología, filosofía, etc.). En su propuesta, ya no es posible contemplar unidades elementales discretas, sino una unidad compleja, un todo que no se reduce a la suma de sus partes. Los elementos no están aislados sino que Bertalanffy muestra cómo éstos participan en una misma realidad, se relacionan con tal de llegar a alcanzar una determinada finalidad. La circularidad estudiada por la cibernética se manifiesta en los sistemas de forma clara ya que los elementos se interrelacionan y se regulan con tal de mantener el sistema. Un aspecto importante, y a menudo olvidado, es que Bertanlaffy (1979) propone la noción de sistema, no como un algo real, ni como una concepción puramente formal sino como una noción que permite situarse en un nivel transdisciplinario. Nos permite concebir, al mismo tiempo, tanto la unidad como la diferenciación, no solamente según la naturaleza de sus objetos sino 
también según los tipos y las complejidades de los fenómenos de asociación y organización. Concebir la realidad desde una perspectiva sistémica no es considerar que todo se relaciona con todo (hay aplicaciones de este tipo) sino analizar la estructura organizacional que permite comprender el funcionamiento y funcionalidad del sistema en un conjunto más amplio.

La teoría cibernética de Wiener y las aportaciones de Bertalanffy caminarán conjuntamente aplicándose a múltiples disciplinas. En el campo de la pedagogía, estas aportaciones comienzan a manifestarse en los años sesenta. Sin embargo, conviene mencionar que la pedagogía cibernética desarrollada en ese momento tomó de la teoría cibernética sus aportaciones como teoría de control, entendiendo éste desde un punto de vista bastante determinista. Un buen ejemplo lo ofrecen los trabajos de Louis Couffignal, pedagogo francés que impulsó los estudios cibernéticos resaltando la importancia de la regulación como clave fundamental de los sistemas. Para Couffignal, la cibernética se define como el "arte de hacer eficaz la acción" $(1969,30)$.

Según Couffignal, la acción educativa se ejerce sobre una región limitada, su duración puede limitarse y su ejecución camina hacia la finalidad propuesta. Bajo este esquema, considera que la importancia de la cibernética reside en diseñar sistemas de actuación y medición de la eficacia alcanzada. Las aportaciones de Couffignal al igual que las de autores como Landa (1972) o Frank y Meder (1976) tuvieron su influencia en el campo de la didáctica y el diseño instruccional.

Las propuestas derivadas de este tipo de pedagogía cibernética no resultan muy diferentes a las elaboradas desde modelos conductistas o tecnológicos. En ésta última línea podemos hallar los trabajos de Felix von Cube (1977) quien determina la figura del pedagogo como un técnico que se sitúa fuera del sistema escolar. Conociendo cuales son las finalidades de éste (determinadas por el sistema social), ha de estudiar la mejor forma de organizar el sistema y las estrategias didácticas que permitan alcanzar dichas finalidades. El pedagogo observa el sistema escolar, se encuentra fuera de él.

En España, la teoría cibernética y sistémica fue introducida por el profesor Alejandro Sanvisens ${ }^{2}$, a finales de los años cincuenta, quien desarrolló una dimensión diferente a las descritas. Desde una perspectiva fundamentalmente teórica, Sanvisens adoptó el modelo sistémico-cibernético para mostrar cómo éste permite explicar los rasgos de la educación destacando así el carácter procesual de la misma, la importancia de los procesos reguladores y la idea de educación como proceso que mejora, que optimiza el funcionamiento del propio sistema. La perspectiva de Sanvisens supone un esclarecimiento del concepto de educación. Nos proporciona una nueva forma de abordarlo introduciendo además nociones como la autoorganización o la retroacción anticipatoria que no habían sido utilizadas en el ámbito educativo por los seguidores de Wiener.

2. SAnvisens, A. (1984): Cibernética de lo bumano (Vilassar de Mar, Oikos-Tau). Como ampliación de la perspectiva desarrollada por Sanvisens, conviene también leer los trabajos de MARTínEz, M. (1986): Inteligencia y Educación (Barcelona, PPU), PUIG, J. (1986): Teoría de la educación. Una aproximación Sistémico-cibernética (Barcelona, PPU) y la obra de CaSTILlejo, J. L. Y Colom, A. J. (1987): Pedagogía Sistémica (Barcelona, Ceac). 
En definitiva, a partir de la pedagogía cibernética desarrollada por Sanvisens se ha podido describir más rigurosamente la educación enfatizando los fenómenos de intercambio de información, y la información utilizada para el propio control del sistema, en un sentido optimizante de adaptación a una situación (feedback), a una finalidad (feedbefore) y al medio (regulación).

\section{LA CIBERNÉTICA DE LOS SISTEMAS OBSERVANTES}

“'reales' llama el burgués sólo a las cosas que todos, o por lo menos la mayoría percibe de modo semejante" (HESSE, H.).

En 1958, Heinz von Foerster efectúa una revisión crítica de la teoría de Wiener, concluyendo que la cibernética que éste había desarrollado introducía cambios importantes pero no suponía una ruptura epistemológica ya que se seguía aplicando el modelo desarrollado por la ciencia por el cual el observador está fuera del objeto (del sistema, en este caso) y es capaz de estudiarlo con objetividad. Von Foerster consideró que la cibernética debía ir más allá y afrontar un nuevo modelo epistemológico en el cual el observador formara parte del sistema estipulando sus propios objetivos, su propio papel dentro del mismo. A partir de ese instante se efectúa una distinción entre la cibernética de primer orden y la cibernética de segundo orden... La pregunta que se hace la cibernética de primer orden podemos formularla de la siguiente forma: ¿dónde están los enlaces circulares en este sistema? Mientras que la cuestión que se plantea la cibernética de segundo orden es: ¿cómo generamos nosotros este sistema a través de la noción de circularidad?

La cibernética de segundo orden ha sido desarrollada por autores como von Foerster, Glasersfeld, Bateson, Prigogine, Maturana y Varela, entre otros, y, en la actualidad muchos de sus principios han quedado también plasmados en la teoría de los sistemas complejos o teoría de la complejidad 3 .

La teoría cibernénica de segundo orden introduce tres ideas fundamentales que modifican los supuestos sobre los que se basa la cibernética de primer orden:

\section{Cuestionamiento del principio de objetividad}

En realidad, la cibernética de segundo orden es una teoría de un carácter fuertemente epistemológico. Se cuestiona la concepción de la ciencia clásica en la cual existe una clara separación entre el sujeto y el objeto de investigación.

Desde un punto de vista epistemológico, la cibernética de primer orden se inscribe dentro de la corriente realista y objetivista que considera que el conocimiento se refiere a una realidad estable, objetiva, existente independientemente a que ésta sea conocida por el hombre. Por el contrario, la epistemología desarrollada por la cibernética de segundo orden se enraíza en la filosofía kantiana y con-

3. Las Teorías de la complejidad utilizan los conceptos básicos de la teoría cibernética de segundo orden desde la perspectiva sistémica. 
sidera que "el mundo de la experiencia, ya se trate de la experiencia cotidiana o de la experiencia de laboratorio, constituye la piedra de toque para nuestras ideas." (Glasersfeld, 1988, 23). En este sentido, el conocimiento no implica una correspondencia con la realidad.

Glasersfeld (1988) utiliza las palabras inglesas "match" (corresponder) y "fit" (encajar) para explicar las diferencias entre ambas posiciones. El realismo considera que existe una correspondencia entre el conocimiento y la realidad mientras que este autor sostiene que el conocimiento supone tan sólo un encaje. De este modo, diferentes conocimientos, significados, interpretaciomes y experiencias pueden encajar en una misma realidad. En definitiva, tal y como afirma Maturana, "el observador se hace en la observación y cuando el ser humano que es el observador muere, el observador y la observación llega a su fin" $(1994,158)$.

Bajo esta concepción, el conocimiento no se puede aceptar como universalmente válido. Las explicaciones científicas sólo son válidas en la comunidad de los observadores que aceptan el criterio de éstas. Desde esta perspectiva, una teoría del universo implica a aquél que describe y por consiguiente, el observador forma parte de del propio sistema.

Este planteamiento introduce aspectos de gran complejidad ya que si el observador está inmerso en la observación es obvio que sus emociones son ineludibles. Por consiguiente, de alguna forma éste debería ser consciente de las mismas y darse cuenta de sus repercusiones.

Este es uno de los problemas que claramente se manifiestan en las terapias familiares sistémicas (D. FrIED, 1994). En los primeros desarrollos de este tipo de terapias, el problema estribaba en cómo estudiar todo el entramado familiar en el que el "paciente" estaba integrado. A esta cuestión ya difícil de resolver, las nuevas terapias sistémicas elaboradas a partir de las aportaciones de von Foerster (1991), incorporan como elemento del sistema al propio terapeuta. En consecuencia, éste debe ser capaz de explicitar su propio papel y los límites de sus acciones.

\section{La construcción de la realidad}

La perspectiva desarrollada por la cibernética de segundo orden es, en definitiva, una perspectiva constructivista. De hecho, autores como von Foerster o Glasersfeld se presentan en la realidad como representantes del contructivismo radical (Glasersfeld 1988; 1994; Von Foerster 1991).

Este tipo de constructivismo es muy similar al desarrollado por Piaget. Desde esta perspectiva cibernética, la construcción es el resultado de la autorregulación del sistema. Para que haya construcción del conocimiento debe haber también un proceso de reflexión. La función de la cognición es adaptativa y sirve para organizar el mundo experiencial del sujeto y no para describir una verdad o realidad ontológica objetiva. El conocimiento es pues como un mapa de senderos de acciones y pensamientos que en el momento de la experiencia se han convertido en viables.

El concepto de viabilidad fue establecido por Glasersfeld (1988) y hace referencia a aquellos conceptos que son útiles para la supervivencia. Según este autor, 
en la praxis, el juicio de una teoría se apoya única y exclusivamente en el hecho de que hasta ahora no ha fracasado. El conocimiento tiene que ser viable, adecuarse a nuestros propósitos. De las teorías se mantienen aquellos conceptos que resultan útiles para la supervivencia. En definitiva, el conocimiento equivale a una función de superviencia y no a una descripción del exterior. Lo que interesa es que los conocimientos que construyo encajen lo suficiente para asegurar su viabilidad.

\section{La complejidad}

Como ya hemos visto, en la cibernética de primer orden el feedback negativo es el mecanismo básico de regulación gracias al cual se produce la organización y regulación del sistema. El feedback positivo mostró cómo en determinados momentos la búsqueda del equilibrio conlleva a una mayor desviación y, por ello, el sistema acaba reestructurándose o creando nuevos patrones de funcionamiento. Los sistemas poseen un alto nivel de complejidad ya que no sólo están influenciados por sus relaciones con otros sistemas sino que tienen también capacidad de autoorganizarse y autoreproducirse.

Un sistema humano, un sistema social no es un sistema de equilibrio. Por el contrario, constantemente se producen perturbaciones, desviaciones que fuerzan a una constante reorganización y ajuste. En este sentido, el orden y el desorden "Cooperan" para la organización del sistema. El desorden es necesario para la producción del orden. Esta relación dialéctica forma parte de la complejidad de los sistemas. Según Morin (1981, 223), los sistemas se desarrollan en un bucle constante formado por:

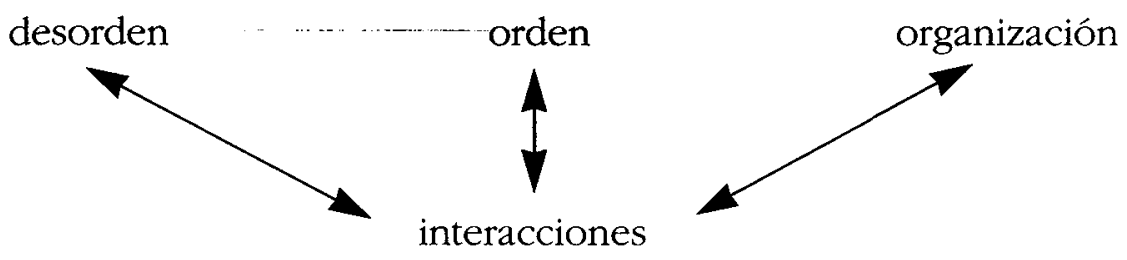

De este modo, percibir la organización de los sistemas desde la complejidad supone incorporar en el análisis sistémico aspectos referidos tanto a la organización y la estabilidad como al desorden y la inestabilidad́t.

En definitiva, la teoría de sistemas propuesta por Bertalanffy se amplia. Los sistemas sociales están formados por elementos que se interrelacionan pero no sólo porque tengan una finalidad común, sino también porque poseen una funcionalidad. Los elementos tienen capacidad de regulación pero también de autorregulación y son sistemas autopoéticos, es decir, pueden autorreproducirse.

4. La utilización de conceptos como azar, estructuras disipativas, fluctuaciones, etc., tiene su origen en los estudios del campo de la física y la biología. En este sentido, recomiendo las lecturas de las obras de PRIGOGine, I. (1983): ¿Tan solo una ilusión? Una exploración del caos al orden (Barcelona, Tusquests) y de WAGENSBERG, J. (1985): Ideas sobre la complejidad del muno (Barcelona, Tusquests). 


\section{LA EDUCACIÓN DESDE LA TEORÍA DE LA COMPLEJIDAD}

"Si el azar no interviniera con su hábil mano en nuestra educación ¿qué sería de nosotros? (LichternBerg. G. CH.).

Como ya hemos mencionado al principio de este artículo, considero que la perspectiva cibernética y sistémica proporciona herramientas conceptuales muy potentes para pensar sobre la educación. He mencionado también que dentro de las diferentes aportaciones de estas teorías considero que la cibernética de segundo orden es la que mejor se ajusta al estudio de la educación. Hasta este momento me he limitado a ofrecer una breve descripción de algunos de los presupuestos básicos de estas posturas. A continuación, intentaré convencer de su importancia para la pedagogía.

En primer lugar, cabe mencionar que durante muchos años se ha discutido sobre la dimensión científica de la pedagogía. Parece obvio que la pedagogía no puede compararse con las ciencias duras y desde esta perspectiva su carácter científico es cuestionable. Como sice von Foerster, "Las ciencias duras tienen éxito porque se ocupan de problemas blandos; las ciencias blandas se les ven en figurillas porque se ocupan de problemas duros" (teorema $\mathrm{n}^{\circ} 2$ de von Foerster, 1991, 179).

Si comparamos a la pedagogía con otras disciplinas más afines tales como la psicología o la sociología parece que aquí la pedagogía también es distinta, es "menos ciencia", es más imprecisa, sus verdades no parecen tan contundentes como las que, por ejemplo, ofrece la psicología. Juzgar las aportaciones pedagógicas desde este punto de vista supone situarse de nuevo en una concepción de lo científico que la pedagogía no puede satisfacer. En el ámbito educativo no hay observadores imparciales, no hay realidades objetivas, no hay determinismo. La educación está compuesta de subjetividades, ideologías, valores, sentimientos, emociones, racionalidades, etc. Por este motivo, lo educativo posee muchos de los rasgos que caracterizan la complejidad de los sistemas. La pedagogía se ocupa de problemas esencialmente no lineales cuyos fenómenos más notorios son las interacciones entre sus partes.

La visión no compleja de las ciencias sociales y de las ciencias humanas ha llevado a una disgregación y simplificación de la realidad. Parece que haya realidades antropológicas, sociológicas, psicológicas, etc. Como si todas estas diversas categorías fueran reales. Sin embargo, a la pedagogía la separación de estas realidades se le hace muy difícil ya que forma parte de ella misma.

El pensamiento pedagógico derivado de una visión analítica y determinista de la educación restringe las formas de conocimiento educativo a la búsqueda de cadenas causa-efecto. La circularidad de los procesos así como los grados de incertidumbre de los mismos son aspectos que no pueden ser obviados.

En la teoría educativa existe un elevado grado de incertidumbre o indeterminación que se manifiesta de forma clara en la práctica. En este sentido, considero que uno de los fracasos más estrepitosos de los planteamientos tecnológicos ha sido la eliminación de estos grados de incertidumbre. Las situaciones predecibles 
junto con la existencia de la incertidumbre, la indeterminación, las situaciones azarosas forman parte de la realidad del sistema educativo. Por ello, estoy totalmente de acuerdo con F. Barcena (1993) quien considera que la educación como tarea reflexiva supone reconocer la existencia de ciertas zonas de incertidumbre en la actividad cuyo control lleva a un gran desgaste reflexivo para cualquier educador profesional.

La introducción de conceptos como incertidumbre, azar, desorden, etc. puede conducir a pensar en la pedagogía como una disciplina imposible. Sim embargo, no es esta la idea que deseo transmitir. Existen también certidumbres o, al menos, como dice Bárcena incertidumbres estructuradas. Es decir, situaciones para las que se poseen conocimientos y recursos apropiados, pero éstos no niegan los no predecibles o simplemente los difusos.

Aunque considero que la teoría cibernética y la teoría de sistemas proporcionan una forma de ver la realidad más completa, no creo que pueda defenderse que la acción se desprenda única y exclusivamente de éstos planteamientos teóricos. El atractivo de los conceptos cibernéticos y sistémicos consiste en su alto nivel de abstracción y en la utilización general que conllevan. Por ello, estoy totalmente de acuerdo con la afirmación de Simon quien considera que "de semejante cambio del ángulo de visión no surgen natural y automáticamente nuevas opciones para nuestras acciones diarias. Pues el peligro ligado a la utilización de tales modelos abstractos consiste precisamente en lo que constituye también su ventaja: su abstracción" $(1994,132)$. En esta misma linea Bateson afirma: "lo que falta es una teoría de la acción dentro de sistemas más amplios y complejos en los cuales el agente activo mismo sea una parte y un producto del sistema" $(1993,325)$.

De hecho el interés en las úlltimas décadas por la investigación de la estructura del pensamiento práctico de los educadores ha llevado a considerar dos aspectos importantes (Bárcena, 1994): En primer lugar, que los profesionales de la educación son agentes racionales, reflexivos y profesionales capaces de reflexionar sobre su práctica. En segundo lugar, que la práctica educativa es una actividad singular, compleja e incierta. El análisis de esta complejidad se encuentra tanto si consideramos la educación como un objeto de conocimiento como si por ella entendemos una actuación o actividad humana.

Este tipo de investigación educativa aunque no se enmarca dentro de la perspectiva sistémica adopta una forma de concebir la educación muy similar a las defendidas por autores como Morin y Bateson.

En definitiva, a pesar de las limitaciones mencionadas, considero que entender la educación desde una perspectiva sistémico-cibernética, proporciona una forma de concebir el funcionamiento de la realidad que ya de por sí conduce a la búsqueda de formas de intervención más completas. La conciencia de la complejidad nos hace comprender que no es fácil escapar de la incertidumbre, pero ello no invalida la construcción de conocimientos viables. 
BEGOÑA GROS SALVAT

PENSAR SOBRE LA EDUCACIÓN DESDE UNA CONCEPCIÓN SISTÉMICO-CIBERNÉTICA

\section{BIBLIOGRAFÍA}

BARCENA, F. (1993): El tratamiento de la incertidumbre en la enseñanza reflexiva. Bases para una teoría del juicio pedagógico, Revista de Educación, 300, pp. 105-132.

BARCElona, F. (1994): La práctica reflexiva en educación (Complutense, Madrid).

Bateson, G. (1979): Mind and Nature. A Necessary Unity (New York, Batam boods).

Bateson, G. (1993): Una unidad sagrada. Pasos ulteriores hacia una ecología de la mente (Barcelona, Gedisa).

Bertalanffy, L (1979): Perspectivas en la teoria general de sistemas (Madrid, Alianza).

Couffignal, L. (1969): La cibernética (Barcelona, A. Redondo).

CuBE, F. (1977): La Ciencia de la Educación (Barcelona, Ceac).

Foerster, H. (1991): Las semillas de la cibernética (Barcelona, Gedisa).

Frank, H.-Meder, B. (1976): Introducción a la Pedagogía Cibernética (Buenos Aires, Troquel).

FRIED, D. (Coord.) (1994): Nuevos paradigmas, cultura y subjectividad (Barcelona, Paidós).

Glasersfeld, E. (1995): A Constructivist Approach to Teaching, pp. 3-16, en STEFFE, L.-Gale, J. (eds.): Constructivism in Education (Hillsdale, Lawrence Erlbaum Associates).

Glasersfeld, E. (1994): Despedida de la objetividad, pp. 19-31, en Watzlawick, P.-Frieg, P. (eds.): El ojo del observador (Barcelona, Gedisa).

GLASERSFELD, E. (1988): Introducción al constructivismo radical, pp. 20-37, en WATZLAWICK, P. (ed.): La realidad Inventada (Barcelona, Gedisa).

Hesse, H. (1978): Lecturas para minutos (Madrid, Alianza).

JDANKo, A. (1990): Une Interpretation Evolucionniste de la Cybernetique, pp. 77-99, Cybernetica, XXXIII: 2.

KARMiloff-Smith, A. e InHELDER, B. (1984): Si quieres avanzar, hazte con una teoría, pp. 307320, en Carretero, M.-García Madruga, J.: Lecturas de Psicología del Pensamiento (Madrid, Alianza).

LANDA, L. (1972): Cibernética y Pedagogía (Barcelona, Labor).

LichternBERG, G. CH. (1990): Aforismos (Barcelona, Edhasa).

Maruyama, M. (1968): The Second Cybernetics: Deviation-Amplifying Manual Causal Processes, en Buckley, W. (ed.): Modern Systems Research for the Behavioral Scienciest, Chicago, Aldine).

Maturana, H. (1994): La ciencia y la vida cotidiana: la ontología de las explicaciones científicas, pp. 157-195, en Watzlawick, P.-Frieg, P. (eds.): El ojo del observador (Barcelona, Gedisa).

Monk, L. (1994): Ludwig Wittgenstein (Barcelona, Anagrama).

MoRín, E. (1994): Introducción al Pensamiento Complejo (Barcelona, Gedisa).

MoRin, E. (1981): El método (Madrid, Cátedra).

Morin, E.-WIENER, N. (1976): Cibernética: necesidad e insuficiencia (Buenos Aires, Cálden).

Simon, S. (1994): Perspectiva interior y exterior. Cómo se puede utilizar el pensamiento sistémico en la vida cotidiana, pp. 132-142, en Watzlawick, P.-Frieg, P. (eds.): El ojo del observador (Barcelona, Gedisa).

WIENER, N. (1960): Cibernética y Sociedad (Buenos Aires, Editorial Sudamericana).

WIENER, N. (1971): Cibernética (Madrid, Guadiana). 